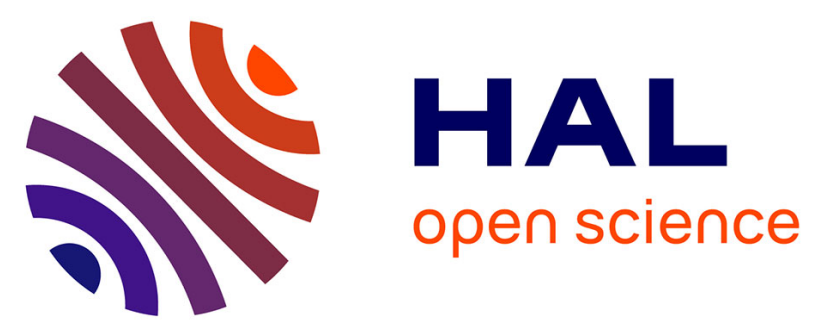

\title{
PRÉVISION DU RAYONNEMENT ACOUSTIQUE DE CYLINDRES FINIS EN PRÉSENCE D'ÉCOULEMENT UNIFORME ET DE SOURCES INTERNES PAR UNE MÉTHODE INTÉGRO-MODALE
}

N. Ouelaa, Bernard Laulagnet, Jean-Louis Guyader

\section{To cite this version:}

N. Ouelaa, Bernard Laulagnet, Jean-Louis Guyader. PRÉVISION DU RAYONNEMENT ACOUSTIQUE DE CYLINDRES FINIS EN PRÉSENCE D'ÉCOULEMENT UNIFORME ET DE SOURCES INTERNES PAR UNE MÉTHODE INTÉGRO-MODALE. Journal de Physique IV Proceedings, 1992, 02 (C1), pp.C1-591-C1-595. 10.1051/jp4:19921127 . jpa-00251083

HAL Id: jpa-00251083

https://hal.science/jpa-00251083

Submitted on 1 Jan 1992

HAL is a multi-disciplinary open access archive for the deposit and dissemination of scientific research documents, whether they are published or not. The documents may come from teaching and research institutions in France or abroad, or from public or private research centers.
L'archive ouverte pluridisciplinaire HAL, est destinée au dépôt et à la diffusion de documents scientifiques de niveau recherche, publiés ou non, émanant des établissements d'enseignement et de recherche français ou étrangers, des laboratoires publics ou privés. 
PREVISION DU RAYONNEMENT ACOUSTIQUE DE CYLINDRES FINIS EN PRESENCE D'ÉCOULEMENT UNIFORME ET DE SOURCES INTERNES PAR UNE MÉTHODE INTÉGRO-MODALE

\author{
N. OUELAA, B. LAULAGNET et J.L. GUYADER \\ Laboratoire Vibrations-Acoustique, BT 303, INSA Lyon, 20 Avenue A. Einstein, \\ F-69621 Villeurbanne cedex, France
}

\begin{abstract}
:
An analytical study of radiation from finite cylindrical shell with infinite rigid extensions is presented.The shell is immersed in an infinite surrounding fluid at rest and contains flowing fluid. The shell is excited either by an harmonic driving force or by internal acoustic sources. The method of resolution consists in expanding the shell wall displacements in series of the simply supported shell eigen modes.

Lntroduction:

L'étude du rayonnement acoustique de cylindres avec présence d'écoulement interne est important en pratique car les tuyauteries sont des sources de bruit dans tous les domaines de l'acoustique industrielle.

Parmi les études existantes dans la littérature, on note l'étude de Gilles F. LEYRAT [3], qui calcule la réponse d'une coque cylindrique mince, de longueur finie, appuyée sur ses bords, excitée par une force ponctuelle, parcourue par un écoulement interne de vitesse constante, sans considération du fluide extérieur. On note aussi les études de C.L. MORFEY [4], FULLER [5], sur la génération et la propagation du son dans une conduite à paroi rigide avec présence d'écoulement interne.

Notre étude, apporte la prise en compte du milieu acoustique extérieur. La sollicitation est soit mécanique ponctuelle appliquée au cylindre, soit acoustique, en particulier une excitation par ventilateur est modélisée par des discontinuités de pression réparties dans une section droite de coque.

Le raccordement avec les études précédentes du L.V.A. (cf. [1], [2]) est possible afin de traiter le cas de coques raidies ou revêtues par des couches élastomère. Enfin, l' application ultime de cette modélisation est le diagnostic des sources internes par analyse de l'acoustique extérieure. (défauts de pâle, etc...).

II Rayonnement acoustlque d'un cylindre bafflé en présence d'un fluide en mouvement at d'un obstacle interne
\end{abstract}

En régime harmonique $\operatorname{Exp}(-j \omega t)$ la pression acoustique à l'intérieur de la coque est régie par les équations suivantes: 
$\Delta P(M)-M^{2} \frac{\partial}{\partial z^{2}} P(M)+2 j K M \frac{\partial}{\partial z} P(M)+K^{2} P(M)=0 \quad M \in V_{i}$

$\left.\frac{\partial P(Q)}{\partial r}\right|_{r=a}=-\rho_{0}\left(-\omega^{2}+V_{z}^{2} \frac{\partial 2}{\partial z^{2}}-2 j \omega V_{z} \frac{\partial}{\partial z}\right) W(Q) \quad Q \in S_{c}$

$\left.\frac{\partial \mathrm{P}(\mathrm{Q})}{\partial \mathrm{r}}\right|_{\mathrm{r}=\mathrm{a}=0} \quad \mathrm{Q} \in \mathrm{S}_{\mathrm{b}}$

$\overline{\mathrm{P}}(\mathrm{Q}) \neq 0 \quad \mathrm{Q} \in \mathrm{S}_{\mathrm{v}}$

$\overrightarrow{\mathrm{V}}(\mathrm{Q}) \neq 0 \quad \mathrm{Q} \in \mathrm{S}_{\mathrm{v}}$

$\overline{\mathrm{P}}(\mathrm{Q})=0 \quad \mathrm{Q} \notin \mathrm{S}_{\mathrm{v}}$

$\overrightarrow{\mathrm{V}}(\mathrm{Q})=0$

$Q \notin S_{v}$

où $\mathrm{V}_{\mathrm{z}}$ est la seule composante non nulle de l'écoulement uniforme, $V_{i}$ le volume interne de la conduite, $S_{c}$ sa surface, $S_{b}$ est la surface du baffle , $M=V_{z} / c$ le nombre de Mach , $c$ la célérité du son , $K=k(1+j \eta)$ le nombre d'onde acoustique complexe, $\eta$ amortisement dans le fluide interne, $W(Q)$ le déplacement radial d'un point $Q$ de la coque , $P(Q)$ la pression acoustique pariétale, po la masse volumique du fluide interne, $\overline{\mathbf{P}}(\mathrm{Q})$ et $\overline{\mathrm{V}}(\mathrm{Q})$ les discontinuités de pression et de vitesse sur $S_{v}$ représentant la surface de l'obstacle.

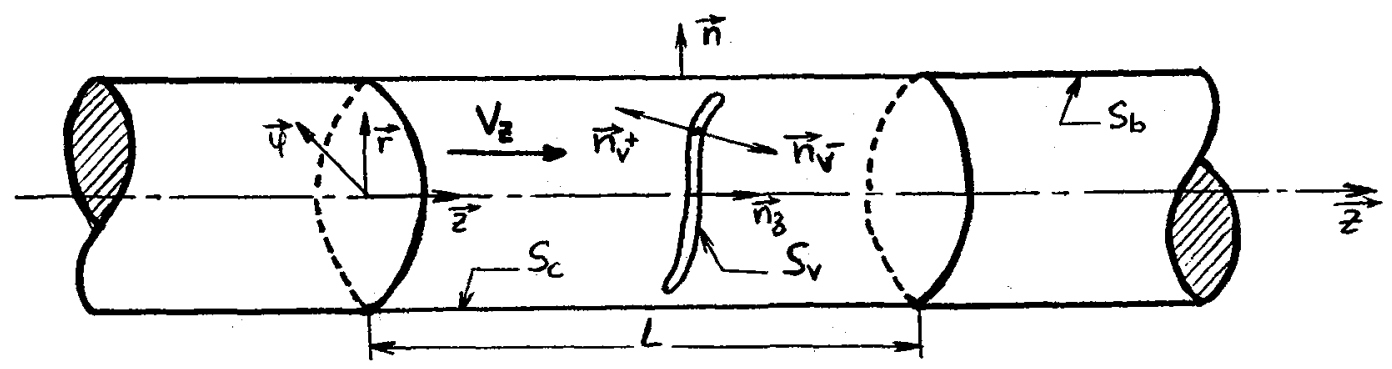

Figure .1. Système de coordonées cylindriques

Pour résoudre le problème de l'acoustique interne, il est utile de définir un problème esclave sous la forme suivante.

$$
\begin{aligned}
\Delta \mathrm{G}\left(\mathrm{M}, \mathrm{Q}_{0}\right)+\mathrm{K}^{2} \mathrm{G}\left(\mathrm{M}, \mathrm{Q}_{0}\right)- & 2 \mathrm{j} \mathrm{KM} \frac{\partial \mathrm{G}\left(\mathrm{M}, \mathrm{Q}_{0}\right)}{\partial \mathrm{z}}-\mathrm{M}^{2} \frac{\partial^{2} \mathrm{G}\left(\mathrm{M}, \mathrm{Q}_{0}\right)}{\partial \mathrm{z}^{2}}=-\delta\left(M-\mathrm{Q}_{0}\right) \\
\left.\frac{\partial \mathrm{G}\left(\mathrm{M}, \mathrm{Q}_{1}\right)}{\partial \mathrm{r}}\right|_{\mathrm{r}=\mathrm{a}}=0 \quad \forall \mathrm{M}_{\mathrm{t}} \mathrm{Q}_{0} \in \mathrm{V}_{\mathrm{i}} & \in \mathrm{S}_{\mathrm{c}} \cup \mathrm{S}_{\mathrm{b}} \text { et } \mathrm{M} \in \mathrm{V}_{\mathrm{i}}
\end{aligned}
$$

$\delta\left(M-Q_{0}\right)$ représente la distribution de Dirac définie dans $R^{3}$ appliquée en $\mathrm{Q}_{0}$.

La résolution du problème esclave précédent nous donne la fonction de Geen définie à droite et à gauche du point $\mathrm{Q}_{0}$ de coordonner $\left(\mathrm{z}_{0}, \varphi_{0}, \mathrm{r}_{0}\right)$. 
$G\left(M, Q_{0}\right)=\sum_{n=-\infty}^{\infty} \sum_{l=1}^{\infty} \frac{j}{2 S} \frac{J_{n}\left(k_{n l} r_{0}\right) J_{n}\left(k_{n l} r\right) \operatorname{Exp}\left(j n\left(\varphi-\varphi_{0}\right)\right)}{\left[1-\left(\frac{n}{k_{n 1} a}\right)^{2}\right] J_{n}^{2}\left(k_{n 1} a\right) \sqrt{K^{2}-\left(1-M^{2}\right) K_{n l}^{2}}}\left\{\begin{array}{l}\operatorname{Exp}\left(j k_{z_{1}}\left(z-z_{0}\right)\right) \\ \operatorname{Exp}\left(j k_{z_{2}}\left(z-z_{0}\right)\right)\end{array}\right\}$

Avec : $\mathrm{k}_{\mathrm{z}_{1}}=\frac{\mathrm{M}+\alpha}{1-\mathrm{M}^{2}} \mathrm{~K}, \mathrm{k}_{\mathrm{z}_{2}}=\frac{\mathrm{M}-\alpha}{1-\mathrm{M}^{2}} \mathrm{~K}, \alpha=\left(1-\left(1-\mathrm{M}^{2}\right)\left(\mathrm{k}_{\mathrm{nl}} / \mathrm{K}\right)^{2}\right)^{-1 / 2}$

$k_{z_{1}}$ et $k_{z_{2}}$ sont les nombres d'ondes définis respectivement à droite et à gauche de $\mathrm{z}_{0}, \mathrm{~J}_{\mathrm{n}}\left(\mathrm{k}_{\mathrm{n}} \mathrm{r}\right)$ est la fonction de Bessel d'ordre circonferentiel $n, k_{n l}$ le nombre d'onde propre des fonctions de Bessel avec condition de Neuman , I ordre radial d'un mode de conduite .

En appliquant le théoreme de Green à $V_{i}$ et en supposant le saut de vitesse nul sur la surface de l'obstacle, la pression interne prend la forme intégrale suivante.

$$
\begin{aligned}
P\left(Q_{0}\right)= & -\rho_{0} \int_{S_{C}}\left(-\omega^{2}+V_{z}^{2} \frac{\partial^{2}}{\partial z^{2}} 2 j \omega V_{z} \frac{\partial}{\partial z}\right) W G d S-\int_{S_{V}} \bar{P} \frac{\partial G}{\partial n_{V}^{+}} d S \\
& +M^{2} \int_{S_{V}} \bar{P} \frac{\partial G}{\partial z} n_{z} d S+2 j K M \int_{S_{V}} \bar{P} G n_{z} d S
\end{aligned}
$$

où $\overrightarrow{n_{v}^{+}}$est la normale à $S_{v}$ (supposée infiniment mince), $\overrightarrow{n_{z}}$ sa projection sur $\overrightarrow{\mathbf{z}}$ (cf. figure 1 ).

L'equation de mouvement de la coque sous la charge de deux fluides, extérieur au repos et intérieur en mouvement uniforme, soumise à une excitation ponctuelle appliquée sur la coque au point $\mathrm{M}_{\mathrm{e}}$ est la suivante.

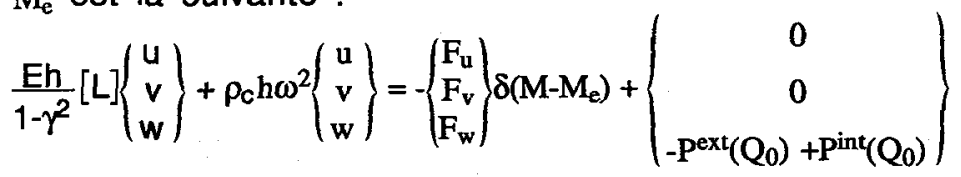

où $(u, v, w)$ sont les composantes du déplacement d'un point de la feuillet moyen de la coque, $\left(F_{u}, F_{v}, F_{w}\right)$ les composantes de la force ponctuelle , $[\mathrm{L}]$ est l'opérateur de DONNELL , $\mathrm{E}$ le module de YOUNG de la coque, $\gamma$ son coefficient de POISSON, $h$ son épaisseur , $\rho_{c}$ sa masse volumique .La détermination de la pression acoustique à l'exterieur de la coque a éte proposée en reference $|6|$, nous y renvoyons le lecteur.

III Décomposition modale et impédance de rayonnement :

La résolution s'effectue par décomposition des fonctions dans la base des modes propres de la coque in vacuo .Les déplacements de la coque sont alors dévoloppés sous la forme suivante. 
$\left\{\begin{array}{c}u \\ v \\ w\end{array}\right\}=\sum_{\alpha}^{1} \sum_{n=0}^{\infty} \sum_{m=1}^{\infty} \sum_{j=1}^{3} A_{n m j}^{\alpha}\left\{\begin{array}{c}D_{n m j} \sin (n \varphi+\alpha \pi / 2) \cos (m \pi z / L) \\ E_{n m j} \cos (n \varphi+\alpha \pi / 2) \sin (m \pi z / L) \\ 1 \quad \sin (n \varphi+\alpha \pi / 2) \sin (m \pi z / L)\end{array}\right\}$

où $L$ est la longueur de la coque entre appuis, $\alpha$ le type de mode ( 0 , antisymetrique ; 1 , symetrique), $n$ l'ordre circonférentiel , $m$ l'ordre longitudinal if le type de mode (flexion, torsion, tractioncompréssion), ( $\left.D_{n m j}, E_{n m j}, 1\right)$ les composantes du déplacement modal, $\mathrm{A}_{\mathrm{nmj}}^{\alpha}$ représente les amplitudes modales du déplacement de la coque Aprés projection des equations (11),(12),(13) dans la base des modes de coque sur appuis, on obtient les equations généralisées du mouvement de la coque suivantes.

$\left\{\mathrm{m}_{\mathrm{pqk}}\left(\omega_{\mathrm{pqk}}^{2}(1-\mathrm{j} \eta)-\omega^{2}\right)\right\} \mathrm{A}_{\mathrm{pqk}}^{\alpha}-\mathrm{j} \omega \sum_{\mathrm{m}=1}^{\infty} \sum_{j=1}^{3}\left(\mathrm{Z}_{\mathrm{pqm}}^{\mathrm{ext}}-\mathrm{Z}_{\mathrm{pqm}}^{\mathrm{int}}\right) \mathrm{A}_{\mathrm{pmj}}^{\alpha}=\mathrm{F}_{\mathrm{pqk}}^{\alpha}-\mathrm{P}_{\mathrm{pq}}^{\alpha}$

Avec : $m_{\mathrm{pqk}}$ masse généralisée , $\mathrm{F}_{\mathrm{pqk}}^{\alpha}$ force généralisée , $\omega_{\mathrm{pqk}}$ pulsation propre,$\eta_{c}$ masse volumique de la coque, $Z_{p q m}^{e x t}$ est l'impédance de rayonnement intermodale qui rend compte du couplage avec le fluide exterieur (cf [6]), zint est l'impédance de rayonnement intermodale qui rend compte du couplage avec structure-fluide interieur en mouvement uniforme, $\mathrm{P}_{\mathrm{pq}}^{\alpha}$ est un terme source due à l'action de l'obstacle interne générant des sauts de pression(par exemple un ventilateur) sur le fluide environnant .

L'expression des impédances de rayonnement directe et croisées du couplage interne sont les suivantes.

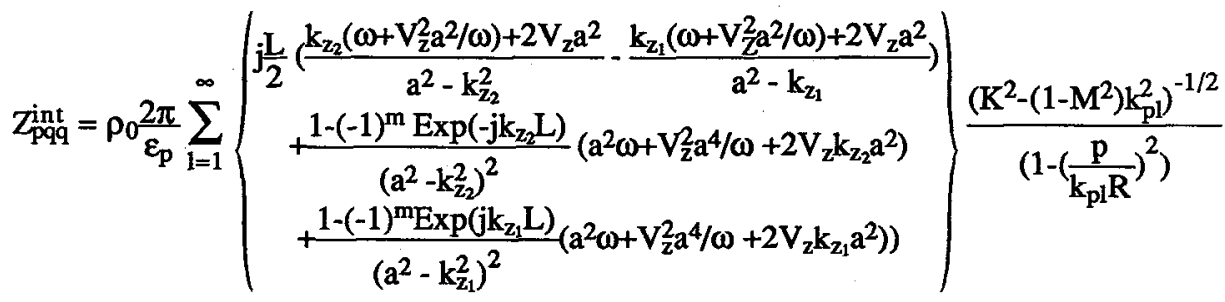

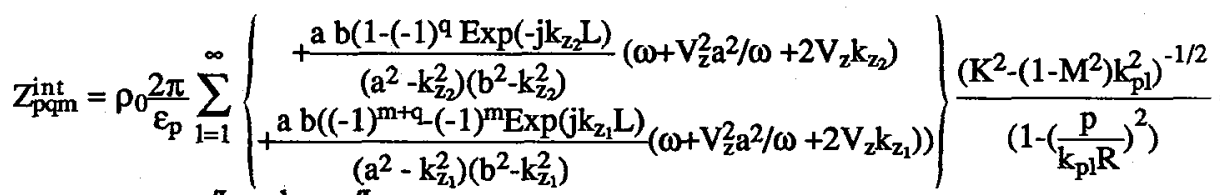

Avec : $a=m \pi / L, b=q \pi / L$

$R$ : est le rayon de la coque , $\varepsilon_{p}=2$ pour $p \neq 0$ et $\varepsilon_{0}=1$, Facteur de Neuman IV Premier résultats:

Nous presentons sur la figure 2 la partie réelle de l'impédance de rayonnement directe avec et sans écoulement pour l'ordre circonferentiel 0 et l'ordre longitudinal 1 . On constate que 
pour des $\mathrm{KL}$ grands les deux courbes tendent vers une valeur asymptotique égale à 0.5 , c'est la même tendance que pour le milieu exterieur voir (cf [6] ). Chaque pic de la figure 2 correspond à un ordre radial I de la section de la conduite. On remarque aussi que l'influence de l'écoulement sur les impedances de rayonnement est trés evident, surtout à base fréquences.

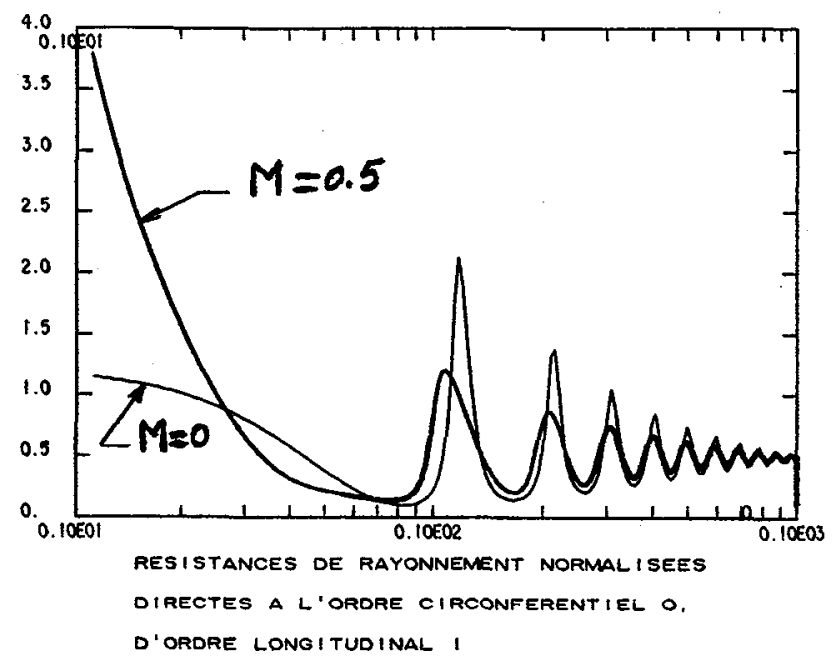

\section{Conclusion :}

\section{Figure 2.}

Dans ce papier nous avons montrés les étapes essentielles de la méthode employée, pour la prévision du comportement vibro-acoustique d'une coque imérgée dans un fluide au repos et contenant un écoulement uniforme. Compte tenu de la place disponible seul un résultat d'impédance de rayonnement interne est donné. Lors de l'exposé une analyse numerique plus approfondie sera présentée.

\section{Bibliographie :}

[1] B. LAULAGNET , J.L. GUYADER , J.S.V. , 1990 , 138(2)

[2] B. LAULAGNET, J.L. GUYADER , A.S.M.E.-J.V.A., 1991, 113

[3] G.F. LEYRAT , Thèse sciences , 1990 , 221 p.

[4] C.L. MORFEY, J.S.V. , 14(1) , 37-35

[5] C.R. FULLER ,D.A. BIES , J.S.V. , 1979 , 62(1) , 73-92

[6] B. LAULAGNET, Thèse sciences , $1989,194 \mathrm{p}$. 